\title{
ESTUDO DAS EMISSÕES OTOACÚSTICAS - PRODUTO DE DISTORÇÃO DURANTE A PRÁTICA ESPORTIVA ASSOCIADA À EXPOSIÇÃO À MÚSICA
}

\author{
Distortion product evoked otoacoustic emissions study \\ with individuals of a fitness gym
}

Isabela Freixo Côrtes-Andrade (1), Aline da Silva de Souza (2), Silvana Maria Monte Coelho Frota ${ }^{(3)}$

\section{RESUMO}

Objetivo: estudar a audição dos alunos de uma academia de ginástica durante o exercício físico, analisando os resultados das emissões otoacústicas - produto de distorção (EOAPD), antes e após a exposição à música eletronicamente amplificada. Métodos: foi aplicado um questionário em 20 indivíduos, sendo os mesmos avaliados por meio das EOAPD, antes e após a execução do exercício físico e exposição à música. Resultados: uma elevada porcentagem de exposições extra-ocupacionais e de lazer (65\%) foi encontrada ao analisar as queixas. Observou-se que $40 \%$ dos alunos de academia de ginástica apresentaram dores de cabeça e insônia; sete, intolerância para sons intensos; cinco, plenitude auricular e irritabilidade; cinco, nervosismo; quatro, tontura; e três relataram apresentar zumbidos após as aulas. No teste de EOPD, houve presença de respostas em $100 \%$ das orelhas testadas no momento 1 (M1), porém, no momento 2 (M2), houve presença de resposta em $75 \%$. As frequências que apresentaram maior percentual de falhas no momento 2 (M2) foram $3 \mathrm{KHz}, 4 \mathrm{KHz}$ e $5 \mathrm{KHz}$. Conclusão: exercício físico associado a elevados níveis de pressão sonora de música provocam alterações nas EOA-PD, principalmente nas frequências de $3000 \mathrm{~Hz}, 4000 \mathrm{~Hz}$ e $5000 \mathrm{~Hz}$. Desse modo, alunos de academia de ginástica que ficam expostos à música eletronicamente amplificada podem estar prejudicando a audição. Há necessidade, portanto, das academias avaliarem as condições acústicas de suas salas de aulas, a partir da análise de profissionais especializados no intuito de verificarem os níveis sonoros, desse modo, observando se estes (NPS) estão compatíveis com os valores recomendados pela lei.

DESCRITORES: Música; Perda Auditiva; Ruído; Audição; Ginástica

(1) Fonoaudióloga da Prefeitura Municipal de Armação de Búzios, Búzios, RJ; Diretora Responsável da Clínica de Fonoaudiologia e Audiologia, AudioNit, Niterói, RJ; Mestre pelo Programa de Estudos Pós-Graduados em Fonoaudiologia da Pontifícia Universidade Católica de São Paulo; Doutoranda do Programa de Estudos Pós-Graduados em Fonoaudiologia da Pontifícia Universidade Católica de São Paulo.

(2) Fonoaudióloga da Associação Fluminense de Reabilitação, AFR, e da Clínica de Fonoaudiologia e Audiologia, AudioNit, Niterói, RJ; Pós-Graduada em Audiologia pela Universidade Castelo Branco no Rio de Janeiro.

(3) Fonoaudióloga; Professora Adjunta do Curso de Fonoaudiologia da Universidade Federal do Rio de Janeiro, UFRJ, Rio de Janeiro, RJ; Professora Adjunta do Curso de Mestrado da Universidade Veiga de Almeida, UVA, Rio de Janeiro, RJ; Doutora em Ciências dos Distúrbios da Comunicação Humana pela Universidade Federal de São Paulo - Escola Paulista de Medicina.

Conflito de interesses: inexistente

\section{INTRODUÇÃO}

Meios de comunicação de massa, como jornais e revistas, têm divulgado em suas matérias diversas discussões e enfoques sobre os transtornos ocorridos com indivíduos expostos a ruídos. O mais interessante é que nem percebemos que convivemos com eles diariamente e que estes se tornam inimigos do organismo.

A música dentro das salas das academias de ginástica é indispensável, pois colabora a tornar agradável o ambiente, bem como a criar uma atmosfera que, no campo subjetivo, seja eficaz no sentido de conforto e bem-estar. Os professores aprendem que quanto mais intensa estiver a música maior o estímulo à atividade física. No entanto, muitas vezes, o fato de que o som excessivamente 
amplificado é prejudicial à saúde é negligenciado na busca por um maior estímulo ao desempenho dos alunos ${ }^{1,2}$.

A música serve para estimular o prazer durante o exercício, proporcionando maior adesão à atividade física e à qualidade de vida. Os estímulos acústicos penetram pela orelha interna e nervo auditivo até o mesencéfalo, por meio do qual o sistema consciente do córtex cerebral entra em estado de alerta elevado (prontidão de reação), estimulando o indivíduo a um melhor desempenho na atividade física. Por sua vez, já é de conhecimento que o aumento excessivo de intensidade pode gerar efeitos nocivos à audição, prejudicando o órgão auditivo ${ }^{3,4}$.

A legislação brasileira afirma que os níveis sonoros que excedem a $85 \mathrm{~dB}(A)$, sejam eles gerados por fones de ouvido, ambiente de trabalho ruidoso, brinquedos sonoros, atividades domésticas e recreacionais, podem acarretar danos à saúde e, principalmente, à audição do indivíduo ${ }^{5}$. De acordo com o Comitê Nacional de Ruído e Conservação Auditiva, a exposição crônica ao ruído acarreta no indivíduo uma deterioração auditiva, lentamente progressiva, caracterizada por perda auditiva neurossensorial, geralmente de grau leve a moderadamente severo; simétrica e irreversível ${ }^{6}$.

As perdas neurossensoriais abrem a possibilidade do surgimento de outros sintomas auditivos, que poderão acompanhar o quadro. Como exemplos desses sintomas estão: zumbidos, dificuldades no entendimento da fala, algicusia, sensação de plenitude dentro do ouvido, sensação de audição abafada e hiperacusia. Outro fator relevante é o efeito extra-auditivo que os indivíduos expostos a níveis de pressão sonora elevado podem apresentar, como ações sobre o aparelho circulatório, digestivo, muscular, sobre o metabolismo, sistema nervoso, interferência no sono, diminuição do rendimento no trabalho, distúrbios de equilíbrio, problemas psicológicos, dores de cabeça, mudanças repentinas de humor e ansiedade 7,8 .

As emissões otoacústicas foram registradas, pela primeira vez, em 1978, por David Kemp. O autor relatou um sinal acústico no meato acústico externo, que parecia um eco da cóclea, após uma estimulação acústica. Após esse estudo, inúmeros outros se sucederam para aprimorar os conhecimentos acerca da existência do mecanismo ativo da cóclea, originado da biomecânica das células ciliadas externas (CCE) do Órgão de Corti. A energia sonora liberada pela cóclea é transmitida de forma reversa pela orelha média até alcançar o meato acústico externo, onde é captada por uma sonda e registrada em um computador ${ }^{9,10}$.

A lesão coclear transitória ou permanente, induzida por exposição à música de alta intensidade, tem sido preocupação de diversos autores 11-18. Estudos têm demonstrado a redução da amplitude de resposta nas EOA, após estimulação acústica $^{19-24}$. Por isso, atualmente, o registro das Emissões Otoacústicas - Produto de Distorção (EOAPD) é a avaliação capaz de identificar as disfunções cocleares iniciais antes de ocorrerem as lesões ocasionadas por exposição ao ruído, beneficiando, pois, no diagnóstico precoce das perdas auditivas ${ }^{25-28}$. Assim, alterações cocleares decorrentes da exposição a níveis elevados de pressão sonora podem provocar precocemente mudanças temporárias na sensibilidade auditiva e redução das amplitudes nas emissões otoacústicas dos produtos de distorção (EOADP).

Um fato agravante é que os alunos das academias de ginástica, na maioria das vezes, preocupam-se com a qualidade de vida e podem já apresentar uma lesão em um órgão tão vital para a comunicação. Tais aspectos justificam o estudo e o controle dos níveis sonoros desta atividade, pois a utilização de métodos de avaliação auditiva que possam detectar perdas auditivas pode colaborar para prevenção e elaboração de programas de preservação auditiva destinados a esses ambientes de lazer.

A partir do exposto, pode ser delineada a hipótese do presente trabalho, isto é: alunos de academias de ginástica constituem uma população de risco para a perda auditiva e devem manifestar queixas extra-auditivas decorrentes da exposição à música excessivamente amplificada em sua atividade profissional?

Frente ao exposto, esta pesquisa terá como objetivo estudar as emissões otoacústicas - produto de distorção, de forma prévia e de forma posterior à exposição a níveis elevados de intensidade durante a atividade física, pesquisando os seus efeitos sobre a audição.

\section{MÉTODOS}

Foi realizado um estudo transversal descritivo, desenvolvido em uma academia de ginástica, localizada na cidade do Rio de Janeiro, no período de março de 2006 a maio de 2007. Obedecendo aos princípios éticos na realização de pesquisas com seres humanos. Todos os participantes receberam uma carta de informação e assinaram um termo de consentimento livre e esclarecido.

Foram avaliados 20 indivíduos (40 orelhas), sendo $8(40 \%)$ do sexo masculino e $12(60 \%)$ do sexo feminino, com idade variando de 20 entre 35 anos, totalizando 40 orelhas. Todos os indivíduos selecionados (100\%) praticavam atividade física, no mínimo, há seis meses. Para tanto, foi aplicado 
um questionário que coletou informações com objetivo de levantar os dados pessoais, de saúde, de exposição à música eletronicamente amplificada, queixas e variáveis que pudessem influenciar os resultados encontrados.

Também foi realizada previamente uma meatoscopia, visando a eliminar quaisquer possibilidades de afecções de orelha externa, como presença de corpo estranho e cerúmen. Somente aqueles que apresentaram ausência de sintomas de doenças otológicas, de rolha de cerúmen e/ou corpo estranho, foram incluídos nessa pesquisa.

Após a meatoscopia, foi aplicado o teste de emissões otoacústica - produto de distorção (EOAPD), realizado em uma sala cedida pela academia de ginástica, com ausência de ruídos, visando a evitar a influência do ruído ambiental durante a aplicação do mesmo. Para a realização do teste foi utilizado o equipamento AuDX da marca BIO-LOGIC SYSTEMS CORP. A relação de intensidade foi de $L 1=$ 65 e L2 = 55dB NPS, proporção de f2/f1 = 1,22 e foi aplicado protocolo "dp-gram" de 0,75 a $8 \mathrm{kHz}$, dois pontos por oitava.

Inicialmente, foram registradas previamente as EOAPD dos alunos selecionados. Em seguida, os alunos se exercitaram na esteira modelo Yozda Fitness Equipament, inclinação plana e velocidade $6 \mathrm{~km} / \mathrm{h}$, por 30 (trinta) minutos, escutando walkman, no volume máximo.

O nível de pressão sonora dos equipamentos foi aferido por meio de um medidor de nível de pressão sonora, o Sound Level Meter - Radio Shack, acoplado na posição $A$, de resposta lenta, no volume máximo dos equipamentos portáteis. A medida da intensidade de saída, usada por eles, nos equipamentos, mostrou valor médio de $90,5 \mathrm{~dB}(\mathrm{~A})$ e os valores de pico em $\mathrm{dB}(\mathrm{A})$ variaram consideravelmente, com valor de pico mais alto em $104 \mathrm{~dB}(\mathrm{~A})$.

Após o exercício, procedeu-se à segunda testagem, com objetivo de comparar os resultados coletados prévia e posteriormente à atividade. Para esse estudo utilizou-se de dois momentos: momento 1 (M1) para os resultados realizados préatividade física; e momento 2 (M2) para os resultados pós-atividade física.

As EOAPD foram consideradas presentes quando a diferença entre a amplitude e o ruído foi acima de pelo menos $6 \mathrm{~dB}$ NPS acima do primeiro desvio padrão do ruído equivalente da frequência avaliada, ou 3dB NPS acima do segundo desvio padrão ${ }^{25}$.

Este estudo foi aprovado pelo Comitê de Ética em Pesquisa do curso de especialização de Audiologia do CEFAC, sob o número de aprovação 024/7.

Todos os resultados foram submetidos à análise estatística. Sendo empregados os testes não-paramétricos de igualdade de duas proporções, Wilcoxon e qui-quadrado para independência. Para complementar a análise descritiva, utiliza-se a técnica de intervalo de confiança para média.

Cabe ressaltar que se definiu para este trabaIho um nível de significância de 0,05 (5\%). Todos os intervalos de confiança construídos ao longo do trabalho foram construídos com 95\% de confiança estatística.

Destaca-se, ainda, que foram utilizados testes e técnicas estatísticos não-paramétricos, porque as condições (suposições) para a utilização dos mesmos, como a normalidade e homocedasticidade (homogeneidade das variâncias), não foram encontradas (principalmente a normalidade) neste conjunto de dados.

\section{RESULTADOS}

\section{a) Resultados da aplicação do questionário:}

Foram investigadas as principais queixas e/ou sintomas auditivos e extra-auditivos dos alunos, conforme demonstra a Figura 1.

Observou-se que, $65 \%$ dos alunos tiveram algum tipo de queixa; enquanto que $35 \%$ não relataram nenhuma queixa.

\section{b) Resultados das emissões otoacústicas - EOAPD:}

Alterações nas EOAPD foram observadas em nove (45\%) indivíduos e EOAPD normais nos 11 $(55 \%)$ restantes.

Houve presença de resposta de $100 \%$ das orelhas testadas no momento 1 (M1), porém, no momento 2 (M2), o estudo apresentou ausência de $25 \%$ das emissões de produto de distorção na população pesquisada.

Na Tabela 1, observa-se a ocorrência dos achados das emissões otoacústicas, antes e depois da exposição ao ruído, sendo possível verificar a porcentagem de falha, no momento 2, para as orelhas direita e esquerda. Observou-se que os indivíduos que falharam, no segundo momento, falharam em todas as frequências $(3 \mathrm{KHz}, 4 \mathrm{KHz}$ e $5 \mathrm{KHz})$ e em ambas as orelhas.

$\mathrm{Na}$ Tabela 2, bem como na Figura 2, observase que os sujeitos falharam muito mais na orelha esquerda $(40 \%)$ do que na orelha direita $(10 \%)$. Essa diferença entre as orelhas foi considerada estatisticamente significante.

$\mathrm{Na}$ Tabela 3, compararam-se, através do teste de Wilcoxon (dados pareados), os momentos M1 versus M2. Verificou-se, então, que somente na frequência de $4 \mathrm{kHz}$ da orelha direita houve diferença estatisticamente significante entre M1 e M2. 


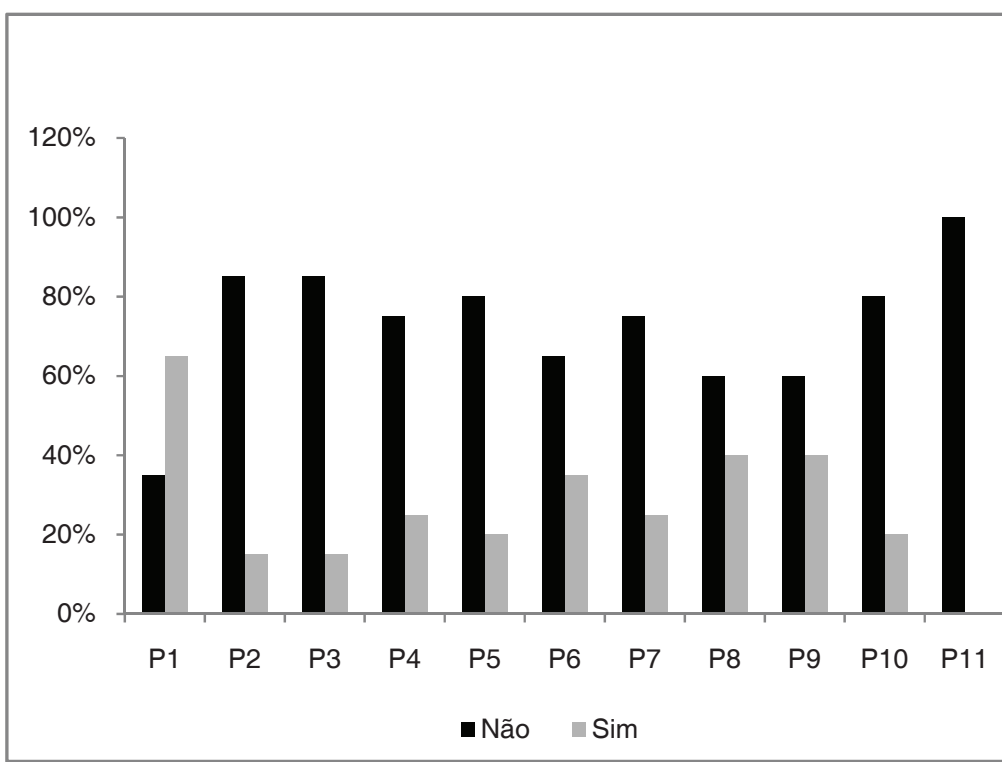

\begin{tabular}{|l|l|}
\hline & Perguntas \\
\hline P1. & Exposições a ruído de lazer? \\
\hline P2. & Dificuldade para escutar? \\
\hline P3. & Zumbido? \\
\hline P4. & Plenitude Auricular? \\
\hline P5. & Trabalha no Ruído? \\
\hline P6. & Intolerância p/som intenso? \\
\hline P7. & Irritabilidade e nervosismo? \\
\hline P8. & Insônia? \\
\hline P9. & Dor de Cabeça? \\
\hline P10. & Tontura? \\
\hline P11. & Outros Sintomas? \\
\hline
\end{tabular}

Legenda: Teste estatístico - Qui-Quadrado.

Figura 1 - Análise dos dados e queixas apresentadas pelos alunos da academia de ginástica

Tabela 1 - Ocorrência dos achados das Emissões Otoacústica - Produto de distorção - antes e após o exercício físico associado a elevados níveis de pressão sonora M1 e M2

\begin{tabular}{|c|c|c|c|c|c|}
\hline \multicolumn{3}{|c|}{ Momentos } & \multirow{2}{*}{$\begin{array}{c}\text { Número de } \\
\text { Orelhas } \\
40\end{array}$} & \multirow{2}{*}{$\begin{array}{c}\begin{array}{c}\text { Porcentagem } \\
\text { De Falha }\end{array} \\
0\end{array}$} & \multirow{3}{*}{$\begin{array}{c}\text { Porcentagem de } \\
\text { Falha na EOAPD } \\
\text { no M2 } \\
10 \%\end{array}$} \\
\hline \multirow{6}{*}{ Orelha Direita } & \multirow{2}{*}{$3 \mathrm{kHz}$} & M1 & & & \\
\hline & & M2 & 40 & 2 & \\
\hline & \multirow{2}{*}{$4 \mathrm{kHz}$} & M1 & 40 & 0 & \multirow{2}{*}{$5 \%$} \\
\hline & & M2 & 40 & 1 & \\
\hline & \multirow{2}{*}{$5 \mathrm{kHz}$} & M1 & 40 & 0 & \multirow{2}{*}{$5 \%$} \\
\hline & & M2 & 0 & 1 & \\
\hline \multirow{6}{*}{$\begin{array}{l}\text { Orelha } \\
\text { Esquerda }\end{array}$} & \multirow{2}{*}{$3 \mathrm{kHz}$} & M1 & 40 & 0 & \multirow{2}{*}{$40 \%$} \\
\hline & & M2 & 40 & 8 & \\
\hline & \multirow{2}{*}{$4 \mathrm{kHz}$} & M1 & 40 & 0 & \multirow{2}{*}{$35 \%$} \\
\hline & & M2 & 40 & 7 & \\
\hline & \multirow{2}{*}{$5 \mathrm{kHz}$} & M1 & 40 & 0 & \multirow{2}{*}{$35 \%$} \\
\hline & & M2 & 40 & 7 & \\
\hline
\end{tabular}

Legenda: Teste estatístico - Não-paramétrico; ${ }^{*} p$-valores: Essa diferença de falha entre as orelhas direita e esquerda não é considerada estatisticamente significante.

Tabela 2 - Ocorrência dos achados das Emissões Otoacústica - Produto de distorção - antes e após o exercício físico associado a elevados níveis de pressão sonora por orelha

\begin{tabular}{cccc}
\hline Falha & Qtde & $\%$ & p-valor \\
\hline Orelha Direita & 2 & $10 \%$ & \multirow{2}{*}{$\mathbf{0 2 8}^{*}$} \\
Orelha Esquerda & 8 & $40 \%$ & \\
\hline
\end{tabular}

Legenda: Teste estatístico - Não-paramétrico; ${ }^{*}$-valores: Essa diferença de falha entre as orelhas direita e esquerda é considerada estatisticamente significante. 


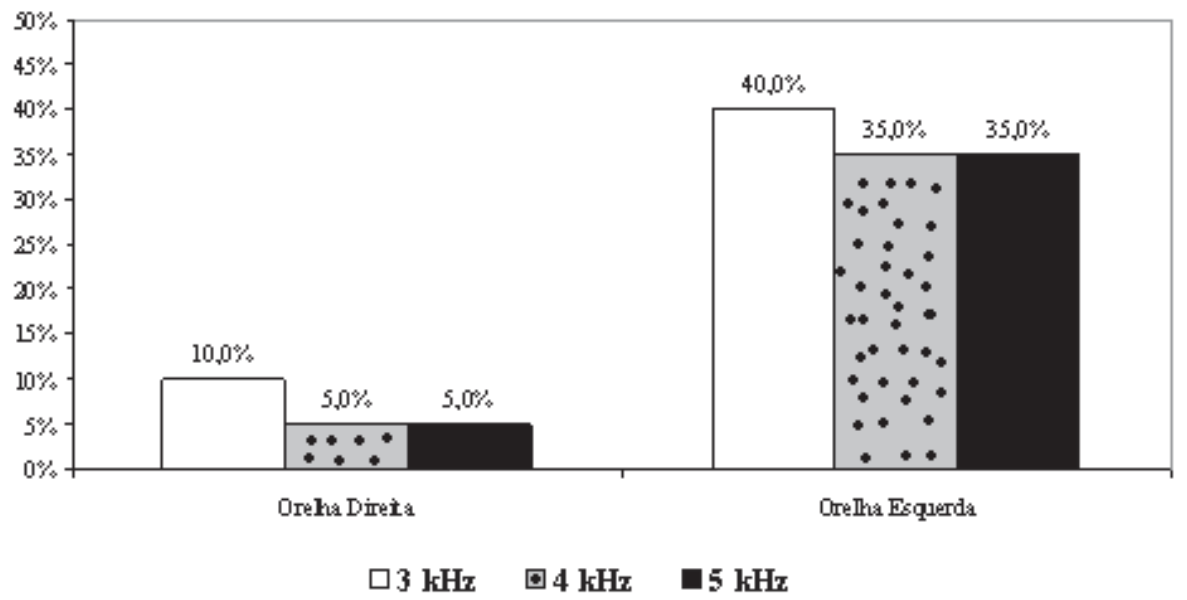

Legenda: Teste estatístico - Não-paramétricos.

Figura 2 - Comparação da relação Sinal x Ruído (S/R) no momento 1 (M1) e no momento 2 (M2) para cada frequência e indivíduo testado

Tabela 3 - Relação sinal ruído no M1 e M2 na orelha direita e na orelha esquerda

\begin{tabular}{|c|c|c|c|c|c|c|c|c|c|c|c|}
\hline \multicolumn{3}{|c|}{ Momentos } & \multirow{2}{*}{$\begin{array}{c}\text { Média } \\
16,39\end{array}$} & \multirow{2}{*}{$\begin{array}{c}\text { Mediana } \\
15,5\end{array}$} & \multirow{2}{*}{$\begin{array}{c}\text { Desvio } \\
\text { Padrão }\end{array}$} & \multirow{2}{*}{$\frac{\text { CV }}{0,30}$} & \multirow{2}{*}{$\frac{\mathbf{Q 1}}{12,5}$} & \multirow{2}{*}{$\frac{\text { Q3 }}{20}$} & \multirow{2}{*}{$\frac{\mathbf{N}}{18}$} & \multirow{2}{*}{$\frac{\text { IC }}{2,28}$} & \multirow{2}{*}{$\begin{array}{c}\text { p-valor } \\
0,420\end{array}$} \\
\hline \multirow{6}{*}{$\begin{array}{l}\text { Orelha } \\
\text { Direita }\end{array}$} & > & M1 & & & & & & & & & \\
\hline & उKHZ & M2 & 14,78 & 16,0 & 5,44 & 0,37 & 10,25 & 18,5 & 18 & 2,51 & \\
\hline & \multirow{2}{*}{$4 \mathrm{kHz}$} & M1 & 17,72 & 18,0 & 6,29 & 0,35 & 12 & 21,75 & 18 & 2,90 & \multirow{2}{*}{$0,035^{*}$} \\
\hline & & M2 & 15,22 & 12,5 & 5,50 & 0,36 & 11 & 21 & 18 & 2,54 & \\
\hline & \multirow{2}{*}{$5 \mathrm{kHz}$} & M1 & 15,72 & 13,5 & 6,66 & 0,42 & 11,25 & 22,75 & 18 & 3,08 & \multirow{2}{*}{0,154} \\
\hline & & M2 & 14,22 & 13,5 & 6,40 & 0,45 & 9 & 19,75 & 18 & 2,96 & \\
\hline \multirow{6}{*}{$\begin{array}{c}\text { Orelha } \\
\text { Esquerda }\end{array}$} & \multirow{2}{*}{$3 \mathrm{kHz}$} & M1 & 16,00 & 17,5 & 4,57 & 0,29 & 13 & 19 & 12 & 2,59 & \multirow{2}{*}{0,694} \\
\hline & & M2 & 17,42 & 18,5 & 5,55 & 0,32 & 13,75 & 22,25 & 12 & 3,14 & \\
\hline & \multirow{2}{*}{$4 \mathrm{kHz}$} & M1 & 16,62 & 17,0 & 4,66 & 0,28 & 14 & 19 & 13 & 2,54 & \multirow{2}{*}{0,755} \\
\hline & & M2 & 16,92 & 16,0 & 6,53 & 0,39 & 14 & 21 & 13 & 3,55 & \\
\hline & \multirow{2}{*}{$5 \mathrm{kHz}$} & M1 & 16,08 & 15,0 & 5,11 & 0,32 & 12 & 19 & 13 & 2,78 & \multirow{2}{*}{0,964} \\
\hline & & M2 & 16,54 & 14,0 & 7,39 & 0,45 & 12 & 18 & 13 & 4,02 & \\
\hline
\end{tabular}

Legenda: Teste estatístico: Não-paramétricos; * $p$-valores: frequência considerada estatisticamente significante; Coeficiente de Variação (CV); $1^{\circ}$ quartil (Q1); intervalo de confiança (IC).

\section{DISCUSSÃO}

Foram encontrados, durante a prática esportiva na academia de ginástica, picos de intensidade que chegaram a $104 \mathrm{~dB}$, ou seja, um valor acima de $85 \mathrm{~dB}$, valor este lesivo e destrutivo ao sistema auditivo, concordando assim com o que relata a legislação brasileira e o Comitê Nacional de Ruído e Conservação Auditiva ${ }^{5,6}$.

Muito tem se discutido quanto à problemática em foco - ruído, poluição sonora e alterações psicofísicas exercidas pelo ruído sobre o ser humano ${ }^{5,6,8,9,20}$. Assim, nos dias atuais, existe a preocupação em se estudar, além dos ruídos profissionais, aqueles ligados ao lazer, procurando-se minimizar os riscos de agressões à orelha interna geradas pelas atividades ruidosas 1,3,4,7,11-16,18,25,29.

Nessa perspectiva, a partir deste estudo, constatou-se que a exposição à música eletronicamente amplificada pode vir a trazer prejuízos extra-auditivos, pois, durante a aplicação do questionário, alguns indivíduos queixaram-se de insônia, zumbido, dificuldade para escutar, dor de cabeça, concordando com o que foi afirmado em alguns artigos ${ }^{1-3,4,7}$.

Do ponto de vista fisiopatológico, a perda auditiva induzida pelo som é essencialmente neurossensorial e, em geral, as Células Ciliadas Externas (CCE) são as primeiras a serem comprometidas ${ }^{5,25}$. Sendo as CCEs as primeiras a serem atingidas na 
exposição à música eletronicamente amplificada, considera-se EOA um exame bastante objetivo para a detecção de perdas auditivas induzidas por níveis de pressão sonora elevados ${ }^{25}$.

Pesquisadores avaliaram o uso das EOA como índice quantitativo da integridade funcional das CCE durante o mecanismo do deslocamento limiar temporário ou Temporary Threshold Shift (TTS), provocado por música de alta intensidade ${ }^{14,29}$. Os autores concluem a utilidade do uso dos produtos de distorção na avaliação e acompanhamento destas alterações, sendo estas mais evidentes nas regiões próximas à frequência de $3.000 \mathrm{~Hz}, 4.000 \mathrm{~Hz}$ e $6.000 \mathrm{~Hz}^{14}$.

Caso as Células Ciliadas Externas (CCE) sejam estimuladas por sons de alta intensidade, a radícula se encurta temporariamente, ocorrendo alterações, em princípio, reversíveis dos cílios ${ }^{10}$. Desde que temporária, a alteração é conhecida como deslocamento limiar temporário (TTS). Da mesma forma, se estimuladas as CCEs com sons mais intensos ou duradouros, havendo lesão celular sem recuperação, estar-se-ia diante do deslocamento limiar permanente (Permanent Threshold Shift - PTS) ${ }^{25}$. Pesquisas afirmam a possibilidade de que repetidos TTS possam levar a um PTS 14,17-20,25.

Por conseguinte, é possível inferir que o tipo de configuração audiométrica decorrente de exposições a níveis elevados de pressão sonora, raramente, atinge o grau de perda profundo, tendo início normalmente nas frequências de $6 \mathrm{kHz}, 4 \mathrm{kHz}$, ou $3 \mathrm{kHz}$, e, com a progressão, atinge as frequências de $8 \mathrm{kHz}, 2 \mathrm{kHz}, 1 \mathrm{kHz}, 500 \mathrm{~Hz}$ e $250 \mathrm{~Hz}{ }^{16,20}$. Verificando os resultados das EOAPD nesta pesquisa, os alunos apresentaram ausência nas frequências de $3 \mathrm{KHz}, 4 \mathrm{kHz}$ e $5 \mathrm{kHz}$.
Portanto, este fato da análise de presença de respostas por frequências, bem como da amplitude das respostas encontradas, concordam com estudos que afirmam que o teste de EOAPD é um importante instrumento de avaliação clínica e acompanhamento dos indivíduos expostos a níveis elevados de pressão sonora de música ${ }^{16,25,26}$.

Como é de conhecimento, a mudança temporária de limiar frequente pode desencadear uma perda auditiva ocupacional. Por esta razão, verifica-se a necessidade de mais estudos com estes profissionais e, também, a implantação de um programa de conservação auditiva.

\section{CONCLUSÃO}

Alunos de academia de ginástica ficam expostos à música eletronicamente amplificada acima do sugerido pelas normas nacionais e, por esta razão, pode-se concluir que são indivíduos de risco para desenvolver perda auditiva induzida por níveis e pressão sonora elevados.

Sugere-se a necessidade das academias avaliarem as condições acústicas de suas salas de aulas e proporem mudanças de hábitos durante a prática esportiva, a partir da análise de profissionais especializados, com o objetivo de verificar os níveis sonoros, assim, observando se estes NPS estão compatíveis com os valores recomendados pela lei.

\section{AGRADECIMENTOS}

Agradecemos ao CEFAC - Pós-Graduação em Saúde e Educação, pelo incentivo e oportunidade de desenvolver esta pesquisa.

\section{ABSTRACT}

Purpose: to study the hearing of individuals in a fitness gym while exercising, analyzing their distortion product evoked otoacustic emissions (DPOAE) test after and before exposure to electronically amplified music. Methods: a questionnaire was applied to 20 individuals and evaluated through DPOAE test after and before physical exercises with electronically amplified music exposure. Results: most of the individuals $(65 \%)$ had extra-occupational exposure. The most frequent complaints were headache and insomnia (40\%); hearing difficulty (35\%); aural fullness (25\%); irritability and nervousness (25\%); dizziness (20\%); and tinnitus (15\%) after exercises. Although DPOAE occurred in $100 \%$ of the ears before the exposure (moment 1 - M1), 25\% failed on the second test, after the exercises (moment $2-$ M2). The frequencies that most failed in the DPOAE test were $3 \mathrm{KHz}, 4 \mathrm{KHz}$ and $5 \mathrm{KHz}$. Conclusion: this study shows that individuals exposed to electronic amplified music, may have hearing loss complaints and have signs of such loss.

KEYWORDS: Music; Hearing Loss; Noise; Hearing; Gymnastics 


\section{REFERÊNCIAS}

1. Deus MJ, Duarte MFS. Nível de pressão sonora em academias de ginástica e a percepção auditiva dos professores. Rev Bras Ativ Fis Saúde. 1997; 2(2):5-16.

2. Palma A, Mattos UAO, Almeida MN, Oliveira GEMC. Nível de ruído no ambiente de trabalho do professor de educação física em aulas de ciclismo indoor. Rev. Saúde Pública. 2009 abr; 43(2):345-51.

3. Martins CO. A influencia da música na atividade física. [monografia]. Florianópolis (SC): Universidade Federal de Santa Catarina; 1996.

4. Amorim RB, Lopes AC, Santos KTP, Melo ADP, Lauris JRP. Alterações auditivas da exposição ocupacional em músicos. Arq Int Otorrinolaringol. 2008 jul-set; 12(3):377-83.

5. Santos JD, Ferreira MIDC. Variação dos limiares audiométricos em trabalhadores submetidos a ruído ocupacional. Arq Int Otorrinolaringol. 2008 abr-jun; 12(2):201-9.

6. Comitê Nacional de Ruído e Conservação Auditiva. Recomendações para a avaliação dos prejuízos ocasionados pela Perda Auditiva Induzida pelo Ruído. Carta aos editores. Acta Awho. 1996; 16:45.

7. Gonçalves MS, Tochetto TM, Gambini C. Hiperacusia em músicos de banda militar. Rev Soc Bras Fonoaudiol. 2007 out-dez; 12(4):298-303.

8. Kasper KCF, Goffi-Gomez MVS, Zaher VL. O ruído como fator estressante na vida de trabalhadores dos setores de serralheria e marcenaria. Rev Arq Otorrinolaringol. 2005, 9(1):48-15.

9. Guedes MC, Passos SN, Goffi-Gomez MVS, Bento RF. Estudo da reprodutibilidade das emissões otoacústicas em indivíduos normais. Rev Bras Otorrinolaringol. 2002; 68(1):34-8.

10. Campos UP, Carvallo RMM. Latência das EOAPD em milissegundos e número de ondas. Rev Bras Otorrinolaringol. 2005; 71(6):784-90.

11. Andrade AIA, Russo ICP, Lima MLLT, Oliveira LCS. Avaliação auditiva em músicos de frevo e maracatu. Rev Bras Otorrinolaringol. 2002; 68(5):714-20.

12. Morata TC. Young people: their noise and music exposures and the risk of hearing loss. Int $\mathrm{J}$ Audiol. 2007; 46(3):111-2.

13. Mendes MH, Morata TC, Marques JM. Aceitação de protetores auditivos pelos componentes de banda instrumental e vocal. Rev Bras Otorrinolaringol. 2007; 73(6):785-92.

14. Pfeiffer M, Rocha RLO, Oliveira FR, Frota S. Intercorrência audiológica em músicas após um show de rock. Rev. CEFAC. 2007 jul-set; 9(3):4239. dx.doi.org/10.1590/S1516-18462007000300017
15. Mendes MH, Morata TC. Exposição profissional à música: uma revisão. Rev Soc Bras Fonoaudiol. 2007; 12(1):63-9.

16. Maia JRF, Russo ICP. Estudo da audição de músicos de rock and roll. Pró-Fono. 2008; 20(1):49-54.

17. Kähäri K, Zachau G, Eklöf M, Möller C. The influence of music and stress on musicians' hearing. J Sound Vibration. 2004; 277(3):627-31.

18. Kähäri K, Axelsson A, Hellström PA, Zachau G. Hearing assessment of classical orchestral musicians. Scand Audiol. 2001; 30(1):13-23.

19. Fiorini AC, Fischer FH. Emissões otoacústicas por transiente evocado em trabalhadores expostos a ruído ocupacional. Dist Comun. 2000; 11(2):167-91. 20. Fiorini AC, Fischer FM. Expostos e não expostos a ruído ocupacional: estudo dos hábitos sonoros, entalhe audiométrico e teste de emissões otoacústicas evocadas por estímulo transiente. Dist Comun. 2004; 16(3):371-83.

21. Barros SMS, Frota S, Atherino CCT, Osteme F. A eficiência das emissões otoacústicas transientes e audiometria tonal na detecção de mudanças temporárias nos limiares auditivos após exposição a níveis elevados de pressão sonora. Rev Bras Otorrinolaringol. 2007; 73(5):592-8.

22. Marques FP, Costa EA. Exposição ao ruído ocupacional: alterações no exame de emissões otoacústicas. Rev Bras Otorrinolaringol. 2006; 72(3):362-6.

23. Shupak A, Tal D, Sharoni Z, Oren M, Ravid $A$, Pratt $H$. Otoacoustic emissions in early noiseinduced hearing loss. Otol Neurotol. 2007 sep; 28(6):745-52.

24. Groh D, Pelanova J, Jilek M, Popelar J, Kabelka Z, Syka J. Changes in otoacoustic emissions and high-frequency hearing thresholds in children and adolescents. Hear Res. 2006; 212(1-2):90-8.

25. Silveira JAM, Brandão ALA, Rossi J, Ferreira LLA, Name MAM, Estefan P, et al. Avaliação da alteração auditiva provocada pelo uso do walkman, por meio da audiometria tonal e das emissões otoacústicas (produtos de distorção): estudo de 40 orelhas. Rev Bras Otorrinolaringol. 2001; 65(5):650-4.

26. Engdahl B, Kemp DT. The effect of noise exposure on the details of distortion product otoacoustic emissions in humans. J Acoust Soc Am. 1996; 99(3):1573-87.

27. Vasconcelos RM, Serra LSM, Aragão VMF. Emissões otoacústicas evocadas transientes e por produto de distorção em escolares. Rev. Bras. Otorrinolaringol. 2008; 74(4):503-7. 
28. Rocha EB, Azevedo MF, Ximenes Filho JA. Estudo da audição de crianças de gestantes expostas ao ruído ocupacional: avaliação por emissões otoacústicas - produto de distorção. Rev. Bras. Otorrinolaringol. 2007; 73(3):359-69.
29. Vinck BM, van Cauwenberge PB, Leroy L, Corthals P. Sensitivity of transient evoked and distortion product otoacoustic emissions to the direct effects of noise on the human cochlea. Audiology. 1999; 38(1):44-52.

DOI: 10.1590/S1516-18462009005000049

RECEBIDO EM: 08/02/2009

ACEITO EM: 24/09/2009

Endereço para correspondência:

Isabela Freixo Côrtes-Andrade

Av. Engenheiro Martins Romeu, 103/802

Niterói - RJ

CEP: 24210-400

E-mail: isabelafono@yahoo.com.br 